\title{
Multiple Fractional Frequency Offset Estimation in LTE System
}

\author{
Dr. C.Geetha Priya \\ Professor, Department of Electronics and Communication Engineering, \\ Kamaraj College of Engineering and Technology, Virudhunagar, Tamil Nadu, India
}

\begin{abstract}
LTE Coordinated Multipoint (CoMP) enables the coordination of transmission and reception over a variety of different base stations. In CoMP-OFDM systems, the multiple transmitters must be synchronized to prevent mutual interference. Applying CoMP transmission, a receiver must estimate multiple CFOs corresponding to the multiple receiving signals for compensation. However, the different signals interfere with each other at the receiver, and this mutual interference significantly degrades CFO estimation performance. Therefore it is difficult to obtain a good estimate of the CFOs. In this paper, fractional CFO in the range of -1 to +1 subcarrier spacing is estimated for multiple base station(BS). The mutual interference and variations to CFO is also minimized.
\end{abstract}

Keywords-long term evolution (LTE); coOrdinated multi point (COMP); maximum likelihood estimator (MLE).

\section{INTRODUCTION}

Orthogonal frequency division multiplexing (OFDM) combined with the CoMP transmission technique is used to improve performance of the receivers located at the cell border. CoMP uses multiple BSs to simultaneously transmit the same information signal. As a result, for a specific user under the CoMP transmission, multiple signals coming from different BSs in the same band are received in the downlink to improve the receiving performance.

Impact of failure in LTE-A network performance will be severe loss, since large investment in small cells has been made in LTE networks to increase capacity and coverage. When synchronization fails, both objectives are lost. Operators need solutions with the built-in capability and flexibility to support synchronization. In the literature, there are excellent work which considered the multiple CFO estimation for coordinated uplink, downlink, multicell, multipath systems. Iterative methods for Inter Carrier Interference (ICI) cancellation and Maximum Likelihood Estimation (MLE) are investigated.

The remainder of the paper is organized as follows : in Section 2, is dedicated to the numerical analysis for the need for CFO estimation and correction in wireless applications like WLAN, WiMAX, LTE etc. Section 3 describe the COMP OFDM System model. Next, the detail algorithm for MLE Multiple CFO is investigated. Section 5 gives simulated results and discussions. The last section of the paper concludes that the estimation range of fractional multiple CFO [-1 1] is achieved accurately with zero mutual interference between BSs.

\section{NEED FOR CFO ESTIMATION AND CORRECTION}

A frequency drift of $10 \mathrm{ppm}\left(10 \times 10^{-6}\right)$ of the local oscillator in a WLAN system with a Carrier frequency of $2.5 \mathrm{GHz}$, results in an offset of $25 \mathrm{kHz}(2.5 \mathrm{GHz}$ X $10 \mathrm{ppm})$. In LTE, which employs a fixed subcarrier spacing of $15 \mathrm{kHz}$, such an offset corresponds to $1.67(25 / 15)$ subcarrier spacings.

Sampling Frequency Offset of 16 parts per million (ppm) of the sampling instant, FFT length of 256, CP length of 64 in WiMAX, received time-domain signal sample will drift $320 \times 16$ ppm samples per OFDM symbol. It will drift one received time-domain complex-valued sample after 195.3125 OFDM symbols. It leads to irreducible intersymbol interference (ISI).

\section{COMP OFDM SYSTEM MODEL}

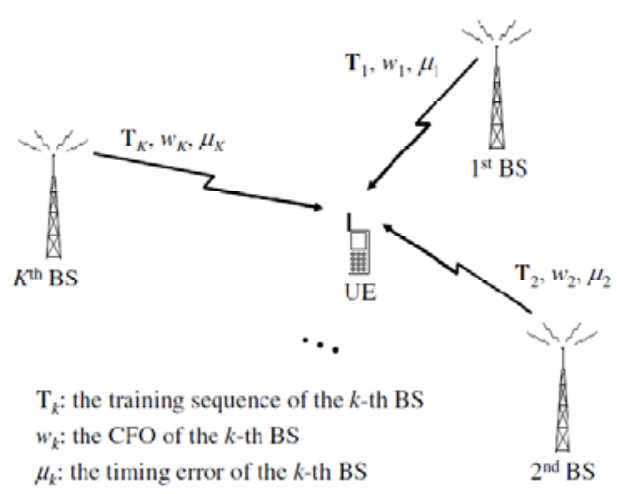

Figure 1. The CoMP OFDM System model [7]

The OFDM system comprises of $\mathrm{N}$ subcarriers. By using CoMP transmission, the signal received at a desired UE is the composite of the signals coming from the $\mathrm{K}$ BSs corresponding to CoMP transmission.

The propagation channel from the $\mathrm{k}$-th $\mathrm{BS}$, for $1 \leq \mathrm{k} \leq \mathrm{K}$, to the UE is an independent $\mathrm{L}_{\mathrm{k}}$-tap multipath fading channel. Let the time-domain discrete signal transmitted by the $\mathrm{k}$-th BS be denoted as $\mathrm{X}_{\mathrm{k}}$ [i], where $\mathrm{i}$ is the time-sample index for $-\mathrm{N}_{\mathrm{cp}} \leq \mathrm{i} \leq \mathrm{N}-1$ and $\mathrm{N}_{\mathrm{cp}}$ is the cyclic prefix (CP) length. After removing the $\mathrm{CP}$, the time-domain discrete signal received at the UE for multi-CFO estimation can be expressed as

$$
\mathrm{y}[\mathrm{i}]=\sum_{\mathrm{k}=1}^{\mathrm{K}} \quad \mathrm{x}_{\mathrm{k}}[\mathrm{i}] \mathrm{e}^{\mathrm{j} 2 \pi \omega_{\mathrm{k}} \mathrm{i} / \mathrm{N}}+v[\mathrm{i}]
$$


where $v[\mathrm{i}]$ is the zero-mean additive white Gaussian noise (AWGN) and $\omega_{\mathrm{k}}$ is the fractional frequency offset obtained as the residual CFO, normalized to the carrier spacing.

$$
v=\left[\begin{array}{lll}
v(0) & v(1) \quad v(N-1)
\end{array}\right]
$$

The received signal in vector form is represented as

$$
y=\left[\begin{array}{lll}
y(0) & y(1) & y(N-1)
\end{array}\right]
$$

The training sequence is the time-domain discrete data transmitted in the synchronization period which can be generated as

$$
Z[i]=\exp \left(j M \pi \mathrm{i}^{2} / \mathrm{N}\right)
$$

for $\mathrm{i}=0,1,2 \ldots . \mathrm{N}-1$

where $\mathrm{M}$ is an integer parameter relatively prime to $\mathrm{N}$.

\section{MLE MultiPLE CFO METHOD}

The received signal in vector form is expressed as

$$
y=\sum_{k=1}^{K} \emptyset_{k} A_{k} h_{k}+v
$$

where $A_{k}$ is the signal matrix, $h_{k}$ is the channel vector and the phase rotation matrix is represented as

$$
\begin{gathered}
\emptyset_{k}=\operatorname{diag}\left(\operatorname { e x p } ( j \varphi _ { k } ( 0 ) ) \ldots \operatorname { e x p } \left(j \varphi_{k}(N-1)\right.\right. \\
\varphi_{k}[i]=2 \pi \omega_{\mathrm{k}} \mathrm{i} / \mathrm{N} \\
A_{k}=\left[\begin{array}{llc}
\mathrm{x}_{\mathrm{k}}[0] & \mathrm{x}_{\mathrm{k}}[1] & \mathrm{x}_{\mathrm{k}}[N-\Delta+1] \\
\mathrm{x}_{\mathrm{k}}[1] & \mathrm{x}_{\mathrm{k}}[0] & \mathrm{x}_{\mathrm{k}}[N-\Delta+2] \\
\mathrm{x}_{\mathrm{k}}[N-1] & \mathrm{x}_{\mathrm{k}}[N-2] & \mathrm{x}_{\mathrm{k}}[N-\Delta]
\end{array}\right]
\end{gathered}
$$

The fractional multiple CFO can be estimated as

$$
\widehat{\omega}=\max \quad \mathrm{y} y^{H}
$$

Where

$$
\widehat{\omega}=\left[\begin{array}{llll}
\widehat{\omega}_{1} & \widehat{\omega_{2}} & \cdots & \widehat{\omega_{\mathrm{k}}}
\end{array}\right]
$$

The objective of designing training sequence set is to apply orthogonal sequences as the training sequences which achieves the zero mutual interference. Constant Amplitude Zero Auto Correlation(CAZAC) are used as the training sequence which have good autocorrelation properties for any non zero circular shifts. Then an orthogonal training sequence for multiple BS's can be generated by circular shifting in time.

$$
Z=[Z(0) Z(1) \quad Z(N-1)]^{T}
$$

Denoting the training sequence as

$$
\left.T_{k}=T_{k}(0) T_{k}(1) \quad T_{k}(N-1)\right]^{T}
$$

is defined for $1<\mathrm{k}<\mathrm{K}$, the training sequence used at the $\mathrm{k}$-th BS as

$$
T_{k}=Z\left\langle D_{k}\right\rangle=\left[Z\left[N-D_{k}\right] \ldots Z\left[N-D_{k}-1\right]\right]^{T}
$$

where $Z\left\langle D_{k}\right\rangle$ is a circular shifting version of $Z$ by right shifting $D_{k}$.

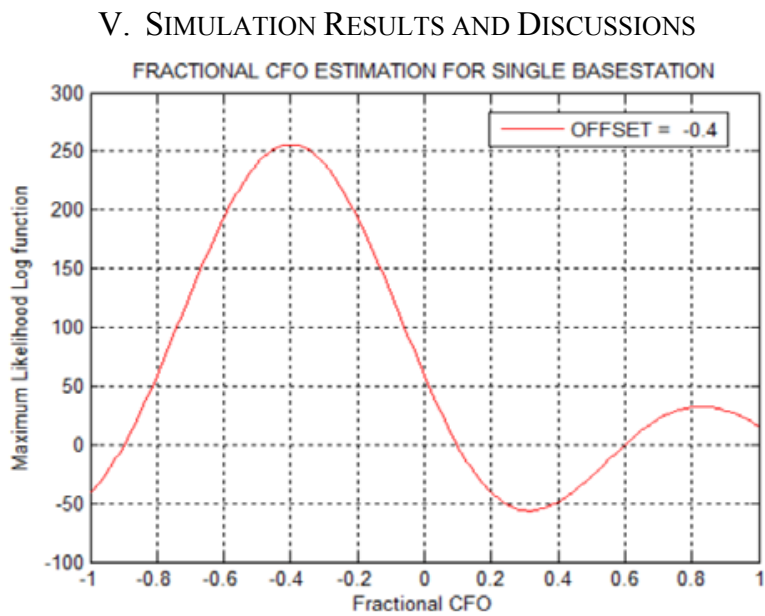

Figure 2. $\mathrm{ML}$ function for a single BS with $\mathrm{CFO}$ of $\omega_{1}=-0.4$

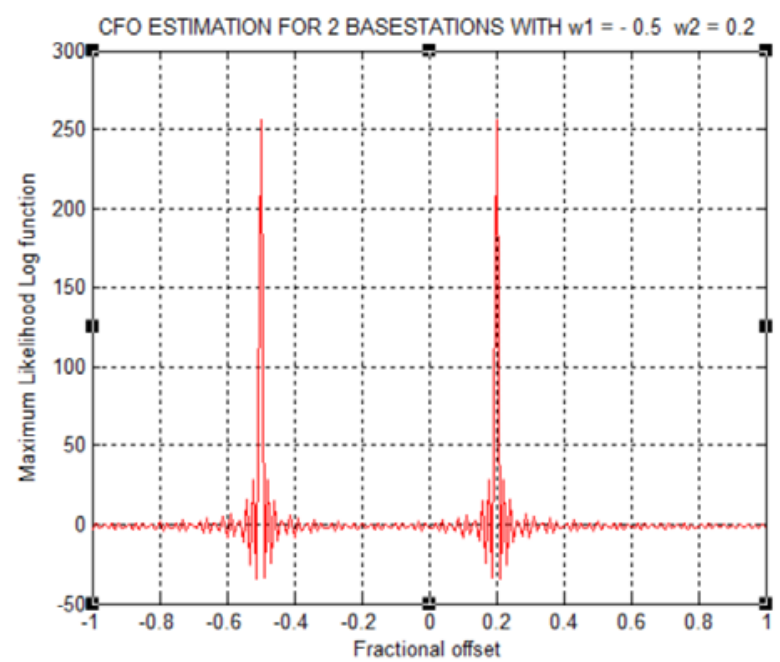

Figure 3. ML function for $2 \mathrm{BSs}$ with $\mathrm{CFO}$ of $\omega_{1}=-0.5, \omega_{2}=0.2$

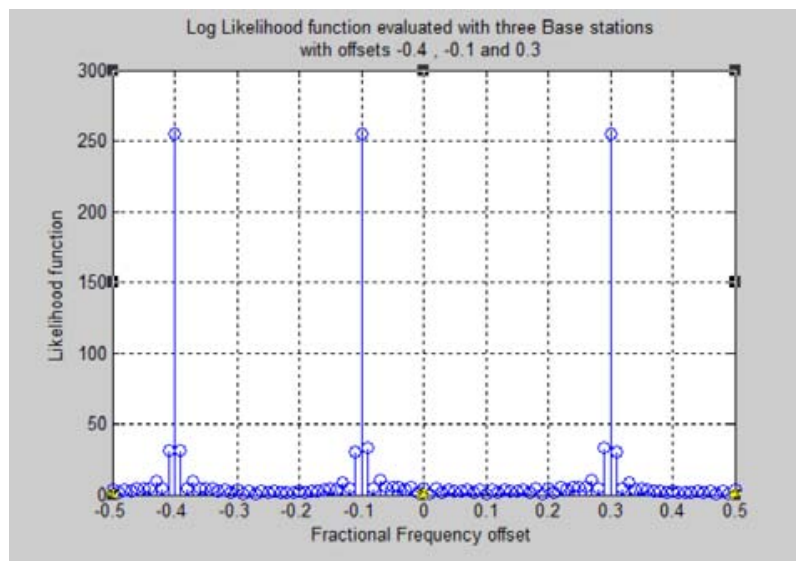

Figure 4. ML function for $3 \mathrm{BSs}$ with CFO of $\omega_{1}=-0.4, \omega_{2}=-0.1, \omega_{3}=0.3$ 


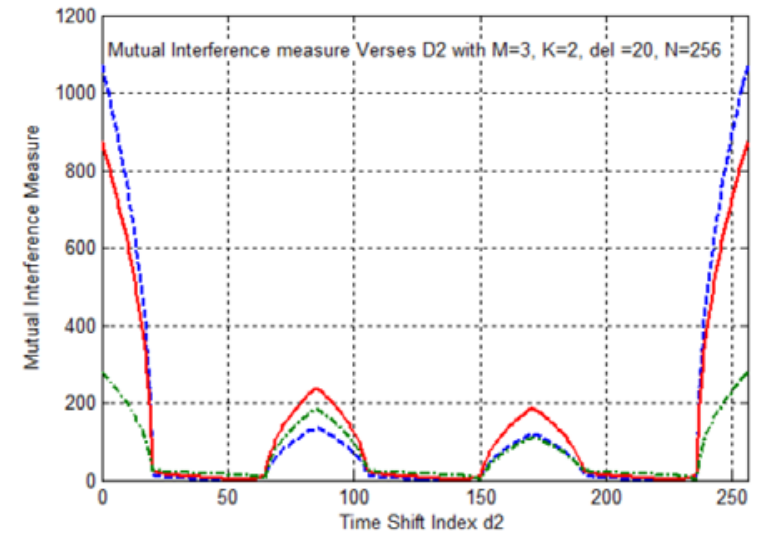

Figure 5. Mutual Interference measure $\mathrm{Vs}_{2}$ with $\mathrm{M}=3, \mathrm{~K}=2, \Delta=20$, $\mathrm{N}=256$

Mutual interference measure $\tau$ Vs $\left(D_{2}, D_{3}\right)$ for $M=3$ and $\mathrm{M}=5$ are calculated using the following expressions

$$
Q(M, q) \equiv\left\lceil\sum_{i=0}^{N-1} \exp \left(-\frac{j 2 \pi i\left[\theta_{1,2}+M_{q}\right]}{N}\right)\right\rceil
$$

$\theta_{1,2}$ is a random variable unknown to the receiver, $\mathrm{q}$ is an integer

$$
\begin{gathered}
\tau\left(M, D_{2}\right) \equiv \sqrt{\sum_{s=-\Delta+1}^{\Delta-1}(\Delta-|s|) Q^{2}\left(M, D_{2}+s\right)} \\
D(\text { opt })=\arg \min (\tau)
\end{gathered}
$$

The $\mathrm{D}_{2}(\mathrm{opt})$ obtained from the simulated results are 43 , $128,213$.

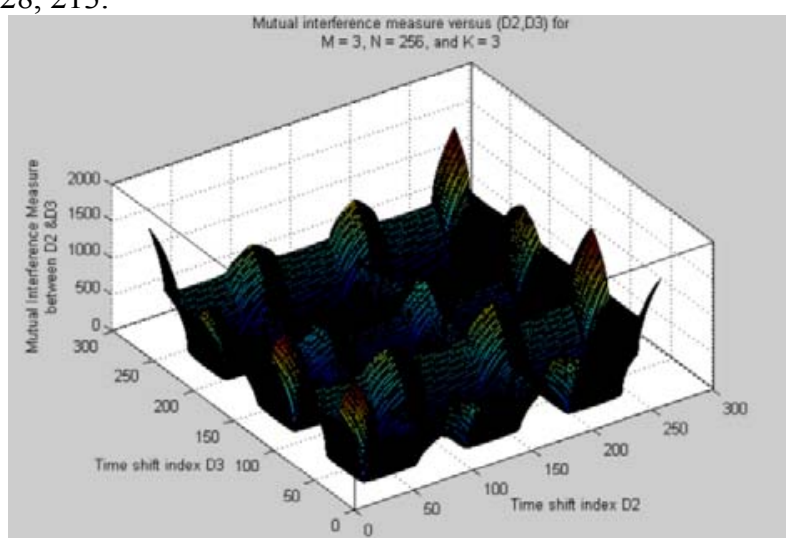

Figure 6. Mutual Interference measure $\mathrm{Vs}\left(\mathrm{D}_{2}, \mathrm{D}_{3}\right)$ with $\mathrm{M}=3, \mathrm{~K}=3, \Delta=20$,

$$
\mathrm{N}=256
$$

The optimal values of the pairs $\left(\mathrm{D}_{2}{ }^{(\mathrm{opt})}, \mathrm{D}_{3}{ }^{(\mathrm{opt})}\right)$ as obtained from the simulation are $(28,57),(28,142)$, $(28,228),(114,57),(114,142),(114,228),(199,57),(199,142)$, $(199,228)$.

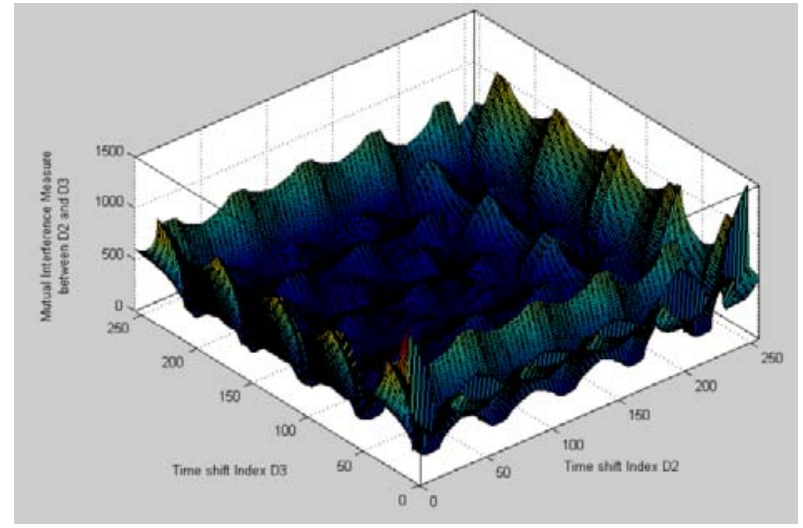

Figure 7. Mutual Interference measure $\mathrm{Vs}\left(\mathrm{D}_{2}, \mathrm{D}_{3}\right)$ with $\mathrm{M}=5, \mathrm{~K}=3, \Delta=20$, $\mathrm{N}=256$.

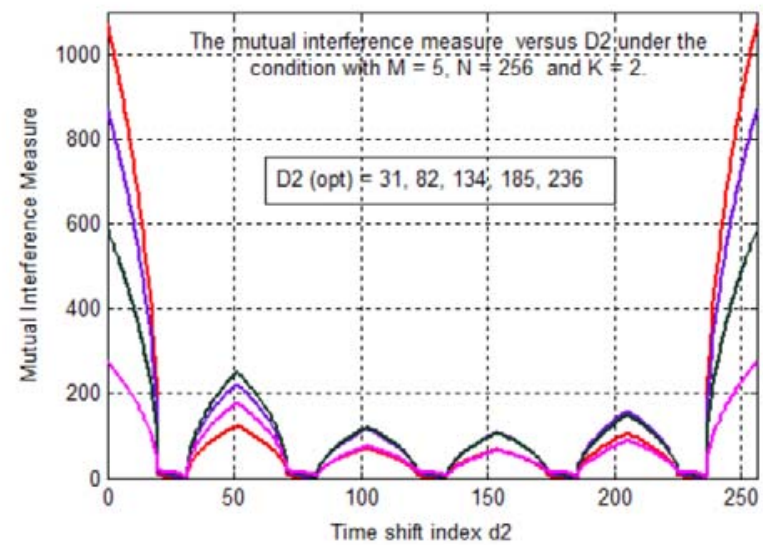

Figure 8. Mutual Interference measure versus D2 with $\mathrm{M}=5, \mathrm{~K}=2, \Delta=20$, $\mathrm{N}=256$

The argument of the peaks yields $\mathrm{D}_{2}$ values with maximum mutual interference.

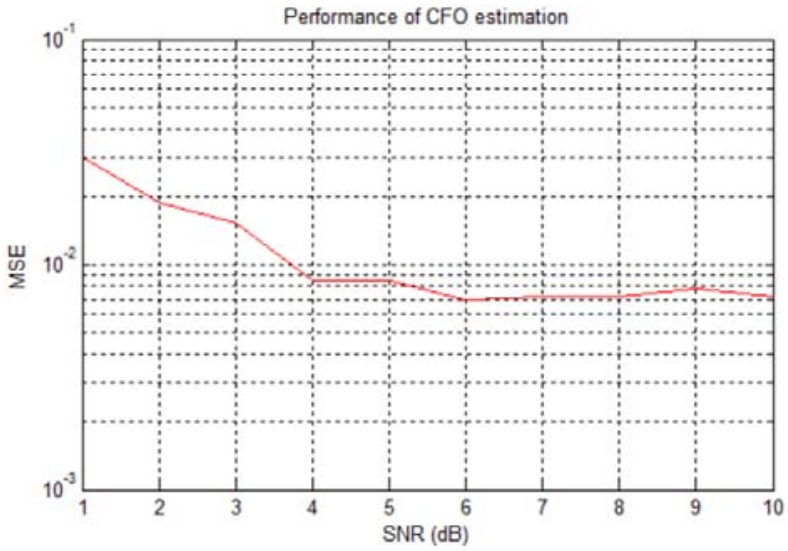

Figure 9. Performance of fractional CFO estimation

\section{CONCLUSIONS}

In this paper, training sequence using CAZAC sequences are designed. The time shift index values for two and more than two base stations are calculated so that there 
is no mutual interference. Simultaneous multiple CFOs in CoMP-OFDM systems is estimated under Rayleigh channels using likelihood function evaluation. The mutual interference measure has been simulated under $\mathrm{M}=5$ and $M=3$ to infer the optimum values of $d_{2}(o p t)$ and the pairs $\left\{\mathrm{d}_{2}(\mathrm{opt}), \mathrm{d}_{3}(\mathrm{opt})\right\}$ for $\mathrm{K}=2$ and $\mathrm{K}=3$ basestations respectively. The performance metric MSE of the algorithm is simulated for various SNR values.

\section{REFERENCES}

[1] Olivier Besson \& Petre Stoica., On Parameter Estimation of MIMO Flat-Fading Channels With Frequency Offsets, IEEE Trans Sig. Proces., 51(3), pp. 602-613, 2003.

[2] Ahamed, S. \& Lam botharan, S., Parameter Estimation for Communication Channels With Multipath and Multiple Frequency Offsets, IEEE Trans. Commun., 53(2),pp. 219-223, 2005.
[3] Brad Zarikoff W. \& James Cavers K., Multiple Frequency Offset Estimation for the downlink of Coordinated MIMO Systems, IEEE Journal on Selected Areas in Communications, 26(6),pp. 901-912, 2008.

[4] Brad Zarikoff, W. \& James Cavers, K., Coordinated Multi-Cell Systems: Carrier Frequency Offset Estimation and Correction, IEEE Journal on Selected Areas in Communications, 28(9), pp. 1490-1501, 2010.

[5] [Yong-Hwa Kim \& Jong-Ho Lee, Joint Maximum Likelihood Estimation of Carrier and Sampling Frequency Offsets for OFDM Systems, IEEE Trans. Broadcasting, 57(2), pp. 277-283, 2011.

[6] Andreas Molisch, F. Martin Toeltsch \& Sameer Vermani, Iterative Methods for Cancellation of Intercarrier Interference in OFDM Systems, IEEE Trans. Vehicular Tech., 56(4),pp. 2158-2166, 2007.

[7] Yuh-Ren Tsai, Hao-Yun Huang, Yen-Chen Chen, \& Kai-Jie Yang, Simultaneous Multiple Carrier Frequency Offsets Estimation for Coordinated Multi-Point Transmission in OFDM , IEEE Trans. on Wireless Commun,12(9),pp. 4558-4568, 2013 